\title{
Réduire la fracture numérique entre les hommes et les femmes par l'éducation
}

Hélène Beaucher

\section{(2) OpenEdition}

1 Journals

Édition électronique

URL : https://journals.openedition.org/ries/9930

DOI : $10.4000 /$ ries. 9930

ISSN : 2261-4265

Éditeur

France Education international

\section{Édition imprimée}

Date de publication : 1 décembre 2020

Pagination : $12-16$

ISBN : 978-2-85420-628-9

ISSN : $1254-4590$

Référence électronique

Hélène Beaucher, "Réduire la fracture numérique entre les hommes et les femmes par l'éducation », Revue internationale d'éducation de Sèvres [En ligne], 85 | décembre 2020, mis en ligne le 01 décembre 2020, consulté le 07 décembre 2022. URL : http://journals.openedition.org/ries/9930 ; DOI : https:// doi.org/10.4000/ries.9930

Ce document a été généré automatiquement le 7 décembre 2022.

Tous droits réservés 


\title{
Réduire la fracture numérique entre les hommes et les femmes par l'éducation
}

\author{
Hélène Beaucher
}

1 Selon les résultats de la dernière enquête internationale ICILS $^{1}$ (International Computer and Information Litteracy Study), qui évalue les performances des élèves de 14 ans en littératie numérique et en pensée informatique, les scores moyens des filles sont supérieurs ou égaux à ceux des garçons dans pratiquement tous les pays participants (https://www.iea.nl/studies/iea/icils/2018).

2 Pourtant, les filles et les femmes sont sous-représentées dans le domaine du numérique, plus encore que dans les filières scientifiques en général ${ }^{2}$.

3 Aujourd'hui dans le monde, les femmes et les filles ont $25 \%$ de chances en moins que les hommes de savoir exploiter les technologies numériques à des fins élémentaires, quatre fois moins de chances de savoir comment programmer un ordinateur et treize fois moins de déposer un brevet technologique ${ }^{3}$. On parle de "fracture numérique entre les genres " pour désigner, dans tous les pays, les différences entre les hommes et les femmes en matière de ressources, de capacités d'accès et d'utilisation efficace des technologies de l'information et de la communication (TIC).

4 Après un état des lieux s'appuyant sur des publications d'institutions internationales, nous proposons dans cet article des rapports et des initiatives qui soulignent le rôle de l'éducation dans la réduction de la fracture numérique entre les genres. Sélection arrêtée le 20 octobre 2020. 


\section{État des lieux de la fracture numérique entre les genres}

\section{Partenariat Equals}

5 Ce rapport du partenariat Equals ${ }^{4}$, une initiative de l'Union internationale des télécommunications (UIT), documente les implications des écarts de genre dans le domaine des technologies numériques dans le monde, en se concentrant sur les trois domaines d'action du partenariat: l'accès aux TIC, les compétences et le leadership. La représentation des femmes demeure faible dans les différents secteurs de l'emploi, de l'entrepreneuriat et de l'élaboration des politiques dans le domaine des TIC. À l'échelle mondiale, 250 millions de femmes de moins que les hommes utilisent l'internet. Les femmes ont globalement moins accès à la téléphonie mobile. Les inégalités de genre concernent les niveaux de compétence les plus bas, l'utilisation d'applications sur un téléphone portable, ainsi que des compétences plus avancées comme le codage. Les principales causes sont liées aux difficultés d'accès aux outils, aux contraintes financières, aux compétences numériques, à l'intérêt pour les TIC, à la sécurité en ligne et aux stéréotypes.

https://www.equals.org/ ; https://bit.ly/332wCkc

\section{OCDE}

6 Réalisé en collaboration avec le G20, un rapport de l'OCDE 2018 intitulé Bridging the digital gender divide: Incluse, upskill, innovate met l'accent sur l'urgence de combler la fracture numérique entre les genres et de renforcer les compétences numériques des femmes. Ces dernières sont sous-représentées dans les emplois des TIC et les hommes ont quatre fois plus de chances que les femmes d'être des spécialistes des TIC. La plupart des facteurs empêchant les femmes de participer pleinement à la société numérique sont basés sur des stéréotypes et des idées préconçues. Ces fractures apparaissent dès le plus jeune âge : à 15 ans, seulement $0,5 \%$ des filles des pays de l'OCDE souhaitent devenir des professionnelles des TIC, contre $5 \%$ des garçons. En outre, les filles sont faiblement scolarisées dans les disciplines qui leur permettraient d'être performantes dans le monde numérique, comme les STEM (acronyme anglais pour sciences, technologie, ingénierie et mathématiques) et les TIC.

https://bit.ly/36XpEOY

\section{Commission européenne}

7 Le rapport Women in the digital age de la Commission européenne ${ }^{5}$, publié en 2018, montre qu'au sein de l'Union, il existe une pénurie de compétences numériques et que celle-ci concerne particulièrement les femmes. Dans l'ensemble de l'Union, les femmes de 16-24 ans sont deux fois moins nombreuses que les hommes de leur âge à avoir appris à programmer. En 2015, 57 \% des diplômés de l'enseignement supérieur étaient des femmes et seulement 24,9 \% d'entre elles étaient diplômées dans les domaines liés aux $\mathrm{TIC}^{6}$; et peu d'entre elles travaillent dans ce secteur. L'étude souligne que l'éducation et la formation doivent être au cœur des stratégies, en mettant en œuvre des mesures spécifiques ciblant différentes tranches d'âge, notamment les jeunes filles 
de 12 à 16 ans, et en adaptant les programmes d'études de l'enseignement supérieur.

https://bit.ly/2UJC2Mp

\section{Recommandations et politiques en éducation}

\section{The role of education and skills in bridging the digital gender divide : Evidence from APEC economies}

Ce rapport 2019 de l'OCDE montre comment l'éducation peut contribuer à réduire la fracture numérique entre les genres, notamment par le développement de l'apprentissage des TIC et de leur utilisation, le renforcement des capacités des enseignants, la modification des attitudes et des attentes et la prévention des discriminations de genre telles que les stéréotypes dans les manuels scolaires. Parmi les solutions proposées : intervenir précocement, encourager les filles à coder, renforcer la participation des filles aux études en mathématiques, en sciences et en informatique, recruter et former des enseignants sensibles au genre et intégrer la culture numérique dans leur formation, veiller à ce que le matériel et les approches pédagogiques soient neutres en termes de genre, mettre en œuvre des programmes de sensibilisation des parents.

https://bit.ly/2V0m28Z

\section{Je rougirais si je pouvais : réduire la fracture numérique entre les genres par l'éducation}

Dans ce rapport rédigé par l'Unesco et le partenariat Equals, le document d'orientation présente des stratégies visant à réduire la fracture numérique entre les genres par l'éducation formelle et informelle, notamment par des approches globales et variées. Les responsables politiques sont invités à intégrer les compétences en TIC dans tous les programmes de toutes les matières et à tous les niveaux d'enseignement et à faire de l'informatique, à l'instar d'un nombre croissant de pays, une matière obligatoire au niveau de l'enseignement secondaire et supérieur. Certains pays ont instauré des conditions d'obtention de diplôme qui nécessitent au moins quelques matières liées aux TIC. Outre les recommandations proposées dans le rapport précédent, citons la mise en œuvre de mesures incitatives, telles que l'octroi de bourses au niveau secondaire et supérieur, le développement de l'apprentissage par projet, collaboratif et par les pairs, la promotion de modèles féminins et le mentorat. Les activités périscolaires et camps thématiques favorisant un apprentissage numérique dans un environnement ludique et détendu sont également recommandés.

https://bit.ly/39gFarF

\section{Initiatives}

\section{La Journée internationale des jeunes filles dans le secteur des TIC}

Mise en œuvre sous l'égide de l'Union internationale des télécommunications, la Journée des jeunes filles dans le secteur des TIC est une initiative mondiale de sensibilisation visant à encourager les jeunes filles et femmes à envisager d'étudier ou 
de faire carrière dans le domaine des TIC. Depuis 2011, cette journée a été célébrée dans 171 pays. Le site suggère des idées d'événements à organiser tels que des ateliers, des journées portes ouvertes dans une institution liée au numérique, des concours, des salons des métiers du secteur des TIC, ou des rencontres professionnelles destinées à montrer que ce secteur n'est pas seulement réservé aux hommes.

https://bit.ly/3lsK2qi

\section{DigiGirlz}

11 Lancée par Microsoft en 2013 et mise en œuvre dans de nombreux pays, l'initiative DigiGirlz vise à sensibiliser au numérique les jeunes filles (principalement les élèves de l'enseignement secondaire supérieur) et à encourager celles-ci à évoluer dans les domaines des technologies. Elle leur permet de s'informer sur les carrières et de participer à des ateliers sur les technologies numériques. L'initiative comprend deux programmes principaux : le "DigiGirlz Day ", un événement d'une journée conçu pour permettre de mieux comprendre à quoi ressemble une carrière dans la technologie, et le «High Tech Camp», un camp de vacances visant à développer les compétences numériques des filles et à les familiariser avec les technologies de pointe, tout en s'efforçant de dissiper les stéréotypes.

https://bit.ly/3lT5pI3

\section{Femmes@Numérique}

12 Créée en 2018, la fondation Femmes@Numérique ${ }^{7}$, qui fédère 45 associations et 42 entreprises, a pour objectif de favoriser la féminisation des métiers du numérique et de mener des actions de sensibilisation des jeunes filles en milieu scolaire, mais aussi de leurs familles, des enseignants, des conseillers d'éducation et plus généralement de tout l'écosystème éducatif. L'un de ses axes de travail consiste à intervenir dans les écoles au travers d'ateliers collaboratifs et de kits pédagogiques, de modèles féminins et de rencontres. Femmes@Numérique finance des actions d'ampleur nationale.

https://femmes-numerique.fr/

\section{Aspirations in Computing (AiC)}

Lancée aux États-Unis par le National Center for Women and Information Technology (NCWIT), cette initiative repose sur un réseau qui œuvre, aux côtés des écoles, à la création d'une communauté durable de femmes. S'appuyant sur les modèles féminins, le mentorat et l'apprentissage par les pairs, le programme de sensibilisation AspireIT de l'AiC enseigne à des filles les rudiments de la programmation au moyen d'activités pratiques, depuis la maternelle jusqu'à la $12^{\mathrm{e}}$ année de scolarité. Sur les 9500 participantes, $75 \%$ se sont déclarées intéressées par un cours d'informatique à l'issue du programme. Au niveau de l'enseignement supérieur, les femmes qui étudient l'informatique comme spécialité ou matière secondaire dans un établissement de l'Alliance académique du NCWIT sont encouragées à rejoindre la communauté AiC. Des bourses, des stages et des offres d'emploi sont également proposés.

https://www.aspirations.org/ 


\section{La Voz de las Chicas del Centro de América}

14 costaricain a été conçu par Sulá Batsú, une coopérative qui vise à encourager et
renforcer le développement local. Mis en œuvre dans les établissements scolaires, il
propose à des filles âgées de 12 à 18 ans, issues de milieux défavorisés, de participer à
des ateliers et à des formations extracurriculaires sur les technologies numériques. Le
projet, étendu désormais à une grande partie de la région, met l'accent sur la musique
numérique, le journalisme communautaire, l'élaboration de prototypes technologiques
et l'apprentissage du code. Il comprend un programme spécifiquement conçu pour les
mères. Chaque formation peut être adaptée aux différentes tranches d'âge et s'attache
à donner aux filles les moyens de trouver des solutions aux problèmes ou aux besoins
qu'elles ont identifiés dans leur communauté. https://bit.ly/3polwsx

\section{NOTES}

1. Organisée par l'IEA (International Association for the Evaluation of Educational Achievement) l'enquête ICILS 2018 a été conduite dans onze pays et deux provinces : Allemagne (Rhénanie du Nord-Westphalie), Chili, États-Unis, Danemark, Finlande, France, Italie, Kazakhstan, Corée du Sud, Luxembourg, Portugal, Russie (Moscou), Uruguay.

2. Voir Minichiello F., "Disciplines scientifiques et inégalités de genre: une préoccupation mondiale", Revue internationale d'éducation de Sèvres, $\mathrm{n}^{\circ} 79,2018$. https:// journals.openedition.org/ries/6787

3. Source : rapport de l'UNESCO et du partenariat Equals, «Je rougirais si je pouvais ", présenté dans cet article.

4. Fondé en 2016 par l'UIT, institution des Nations unies spécialisée dans les TIC, l'ONU Femmes, le Centre du commerce international, GSMA et l'Université des Nations unies, le partenariat mondial Equals vise à réduire la fracture numérique entre les hommes et les femmes et à combler ce fossé d'ici 2030.

5. La Commission européenne publie un tableau de bord annuel, Women in Digital (WID) permettant de suivre la participation des femmes à l'économie numérique. https://bit.ly/ 3 PQRKDN

6. Selon les données d'Eurostat, en 2018, sur les 1,3 million de personnes entreprenant des études supérieures en TIC, les femmes ne représentaient que $17 \%$. https://bit.ly/3nQ7gOt

7. Femmes@Numérique est portée par six associations fondatrices: l'AFMD (Association française des managers de la diversité), la Conférence des grandes écoles, le Cigref / réussir le numérique, Social Builder, Synthec numérique et Talents du numérique. 
INDEX

Mots-clés : numérique, compétences numériques, TIC : technologies de l'information et de la communication, inégalité de genre, femme

Keywords : digital, digital skills, ICT: information and communication technologies, gender inequality, women

Index géographique : Pays de l'OCDE, Union européenne, États-Unis, Amérique latine, France Palabras claves : digital, competencias digitales, TIC: tecnologías de la información y la comunicación, desigualdad de género, mujer

\section{AUTEUR}

\section{HÉLÈNE BEAUCHER}

Hélène Beaucher est documentaliste au Centre de ressources et d'ingénierie documentaires (CRID) de France Éducation international (CIEP). Courriel : beaucher[at]france-educationinternational.fr 